VoL. 45 (1992) [267-276]

\title{
MODIFIED HERMITE POLYNOMIALS IN THE SPECTRAL APPROXIMATION FOR BOUNDARY LAYER PROBLEMS
}

\author{
Nevenka AdžIć
}

\begin{abstract}
The solution of the linear boundary layer problem is presented as a sum of the reduced solution and the layer function, which is approximated by a truncated orthogonal series. The layer subinterval is determined through the numerical layer length, which depends on the perturbation parameter and the degree of the spectral approximation. Modified Hermite polynomials are used as an orthogonal basis. Systematic methods are presented to obtain the recurrence relations for the coefficients in modified Hermite series solution of linear differential equations with polynomial coefficients. The upper bound for the error function is constructed and the numerical example is included.
\end{abstract}

\section{INTRODUCTION}

In this paper we shall consider the boundary layer problem

$$
\begin{aligned}
& L y \equiv-\varepsilon^{2} y^{\prime \prime}(x)+f(x) y^{\prime}(x)+g(x) y(x)=h(x), \quad x \in[0,1] \\
& G y \equiv(y(0), y(1))=(A, B)
\end{aligned}
$$

where $\varepsilon>0$ is a small parameter. Our assumptions are:

$$
\begin{aligned}
& f(x), g(x), h(x) \in C[0,1], \\
& 0<F \leqslant f(x), \quad F \in R, \quad x \in[0,1], \\
& K^{2} \leqslant g(x), \quad K \in R, \quad x \in[0,1] \text {. }
\end{aligned}
$$

It is well known that the solution of the problem (1.1), (1.2) has a boundary layer at $x=1$. The problems of this kind are involved in mathematical models of diffusion-convection phenomena. In many applications the diffusion coefficient $\varepsilon$ is much smaller than the convection coefficient $f(x)$. It has long been recognised that difficulties can arise when standard spectral approximations are applied. The author has already developed the modification of these standard spectral methods in several papers; for example see [5]. Here, that modification is combined with the use of special orthogonal basis, that is, modified Hermite polynomials. The recurrence relations,

Received 2 April 1991

Copyright Clearance Centre, Inc. Serial-fee code: 0004-9729/92 \$A2.00+0.00. 
which are going to be constructed, are much simpler than the corresponding relations for Chebyshev orthogonal basis, obtained by Horner in [2], or for Legendre polynomials, developed by the author and applied in [4]. The method presented here is highly accurate although only a small number of terms in the appropriate truncated series is used. This is confirmed by the numerical results.

In Section 2 we shall transform the original problem and determine the layer subinterval. In Section 3 we shall define the orthogonal projection according to the modified Hermite polynomial basis and we shall develop certain recurrence relations. In Section 4 we shall estimate the error by constructing the upper bound function upon the layer subinterval and in the final section we shall illustrate all the theoretical results by a numerical example.

\section{TRANSFORMation}

It is well known (see for example [1]) that under the assumptions (2.1) - (2.3) the problem (1.1), (1.2) is inverse monotone, which ensures that it has a unique solution $y(x) \in C^{2}[0,1]$. It is also known that the reduced problem

$$
L_{r} y_{r} \equiv f(x) y_{r}(x)+g(x) y_{r}(x)=h(x), \quad x \in[0,1], \quad y_{r}(0)=A
$$

has a unique solution $y_{r}(x) \in C^{2}[0,1]$, which in general, does not satisfy the rightside boundary condition. We are going to look for the solution of (1.1), (1.2) in the form

$$
y(x)=y_{r}(x)+y_{\varepsilon}(x)
$$

where $y_{e}(x)$ is approximated by

$$
\widetilde{y}(x)= \begin{cases}0 & x \in[0,1-\delta] \\ u(x) & x \in[1-\delta, 1]\end{cases}
$$

and $u(x)$ satisfies the boundary value problem

$$
\begin{aligned}
L u & \equiv-\varepsilon^{2} u^{\prime \prime}(x)+f(x) u^{\prime}(x)+g(x) u(x)=\varepsilon^{2} y_{r}^{\prime \prime}(x), \quad x \in[1-\delta, 1] \\
D u & \equiv(u(1-\delta), u(1))=\left(0, B^{0}\right), \quad B^{0}=B-y_{r}(1) .
\end{aligned}
$$

Here $\delta>0$ is the so-called numerical layer length which is going to be determined using the definitions and lemmas, given by the author in several papers, for example [5].

Definition 1: A function $p(x) \in C^{2}[1-\delta, 1]$ is called a resemblance function for the problem (4.3), (4.4) if

$$
\begin{array}{ll}
1^{0} & D p=\left(0, B^{0}\right), \\
2^{0} & x=1-\delta \text { is the stationary point for } p(x), \\
3^{0} & p(x) \text { is concave for } B^{0}>0 \text { and convex for } B^{0}<0 .
\end{array}
$$


LEMma 1. The $n$-th degree polynomial

$$
p_{n}(x)=B^{0}\left(\frac{x-1}{\delta}+1\right)^{n}, \quad n \geqslant 2
$$

is a resemblance function for the problem (4.3), (4.4).

Proof: We have to verify the conditions from Definition 1 . It is easily seen that

$$
\begin{array}{ll}
1^{0} & p_{n}(1-\delta)=0 \text { and } p_{n}(1)=B^{0} \\
2^{0} & p_{n}^{\prime}(x)=0 \quad \text { only for } x=1-\delta \\
3^{0} & \operatorname{sgn} p_{n}^{\prime \prime}(x)=\operatorname{sgn} B^{0}
\end{array}
$$

DEFINITION 2: The sufficiently small number $\delta$, for which the resemblance function satisfies equation (4.3) at the layer point $x=1$, is called the numerical layer length.

Lemma 2. The numerical layer length is given by the expression

$$
\delta=\frac{r n-\sqrt{r^{2} n^{2}-4 q \varepsilon^{2} n(n-1)}}{2 q}
$$

with

$$
q=\frac{\varepsilon^{2} y_{r}^{\prime \prime}(1)}{B^{0}}-g(1), \quad r=f(1)
$$

Proof: By substituting (5) into (4.3), at $x=1$ we obtain the equation

$$
\left(\frac{\varepsilon^{2} y_{r}^{\prime \prime}(1)}{B^{0}}-g(1)\right) \delta^{2}-f(1) n \delta+\varepsilon^{2} n(n-1)=0
$$

and its solution $0<\delta<1$ is given by (6.1). The existence of this solution is ensured by the conditions $(2.1)-(2.3)$ since

$$
D=f^{2}(1) n^{2}-4 \varepsilon^{2} n(n-1)\left(\frac{\varepsilon^{2} y_{\tau}^{\prime \prime}(1)}{B^{0}}-g(1)\right) \geqslant 0
$$

for sufficiently small $\varepsilon$.

Once the numerical layer length $\delta$ is determined we can proceed to construct the approximate solution for the problem (4.3), (4.4). One of the main problems is the approximation of the functions $f(x)$ and $g(x)$. If they are approximated by power series 
or some orthogonal series of large degree, the calculations may be rather complicated. Under the additional assumption (which is not very restrictive) that $f(x), g(x) \in$ $C^{3}[1-\delta, 1]$ it is sufficient to approximate them by the polynomials of degree up to 2 .

$$
f(x) \approx c_{1} x^{2}+c_{2} x+c_{3}, \quad g(x) \approx c_{4} x^{2}+c_{5} x+c_{6} .
$$

REMARK. The order of such an approximation is $O\left(\delta^{3}\right)$, and, as $\delta$ is always small, this approximation does not affect the accuracy of the approximate solution significantly.

As for the function $y_{r}^{\prime \prime}(x)$, it has to be approximated by the appropriate orthogonal series $r_{n}(x)$. Thus, we are looking for the spectral solution of the problem

$$
\begin{aligned}
L_{a} u_{a} \equiv-\varepsilon^{2} u_{a}^{\prime \prime}(x)+ & \left(c_{1} x^{2}+c_{2} x+c_{3}\right) u_{a}^{\prime}(x)+\left(c_{4} x^{2}+c_{5} x+c_{6}\right) u_{a}(x)=r_{n}(x), \\
x & =[1-\delta, 1]
\end{aligned}
$$

$$
D u_{a} \equiv\left(u_{a}(1-\delta), \quad u_{a}(1)\right)=\left(0, B^{0}\right) .
$$

\section{SERIES SOLUTION}

The spectral solution of the problem (8.1), (8.2) is a truncated orthogonal series of degree $n$, according to some orthogonal basis of the space $P^{n}$ of all real polynomials of degree up to $n$. In this paper we are going to choose modified Hermite polynomials as the elements of the basis. Let us, first, remind ourselves of some of their properties.

Classical Hermite polynomials represent a particular solution of the Hermite differential equation

$$
H_{k}^{\prime \prime}(x)-2 x H_{k}^{\prime}(x)+2 k H_{k}(x)=0 .
$$

The modified Hermite polynomials, introduced in [3], are defined as

$$
\begin{aligned}
& \widetilde{H}_{k}(x)=\alpha_{k} H_{k}(\beta x), \\
& \alpha_{k}=\frac{1}{(2 \beta)^{k} k !}, \quad \beta \in R .
\end{aligned}
$$

They satisfy Bonnet's recurrence relation

$$
x \widetilde{H}_{k}(x)=(k+1) \widetilde{H}_{k+1}(x)+\frac{1}{2 \beta^{2}} \widetilde{H}_{k-1}(x),
$$

and for the derivatives we have

$$
\widetilde{H}_{k}^{\prime}(x)=\widetilde{H}_{k-1}(x), \quad \widetilde{H}_{k}^{\prime \prime}(x)=\widetilde{H}_{k-2}(x) .
$$


Our aim is to approximate the solution $u_{a}(x)$ of the problem $(8.1),(8.2)$ by

$$
u_{n}(x)=\sum_{k=0}^{n} a_{k} \tilde{H}_{k}(x)
$$

and it is well known that when $n \rightarrow \infty, u_{n} \rightarrow u_{a}$. If we represent $r_{n}(x)$ as

$$
r_{n}(x)=\sum_{k=0}^{n} b_{k} \widetilde{H}_{k}(x)
$$

we can prove the following theorem:

THEOREM 1. The coefficients $a_{k}$ in the solution (11) of the problem (8.1), (8.2) represent the solution of the system

$$
\sum_{\substack{i=k-2 \\ i \geqslant 0}}^{k+3}\left(\sum_{j=0}^{6} c_{j} \omega_{i, j}^{(k)}\right) a_{i}=4 \beta^{4} b_{k}, \quad k=0,1, \ldots, n-2, \quad c_{0}=\varepsilon^{2}
$$

$$
\sum_{k=0}^{n} a_{k} \widetilde{H}_{k}(1-\delta)=0, \quad \sum_{k=0}^{n} a_{k} \widetilde{H}_{k}(1)=B^{0}
$$

where $\omega_{i, j}^{(k)}$ is the element of the $i$-th row and $j$-th column of the matrix $W^{(k)}$, the non-zero elements of which are given in Table 1.

Table 1.

\begin{tabular}{|l|c|c|c|c|c|c|c|}
\hline & $\varepsilon^{2}$ & $c_{1}$ & $c_{2}$ & $c_{3}$ & $c_{4}$ & $c_{5}$ & $c_{6}$ \\
\hline $\begin{array}{l}i=k+3 \\
a_{k+3}\end{array}$ & & 1 & & & & & \\
\hline $\begin{array}{l}i=k+2 \\
a_{k+2}\end{array}$ & $-4 \beta^{4}$ & & $2 \beta^{2}$ & & 1 & & \\
\hline $\begin{array}{l}i=k+1 \\
a_{k+1}\end{array}$ & & $2 \beta^{2}(2 k+1)$ & & $4 \beta^{4}$ & & $2 \beta^{2}$ & \\
\hline $\begin{array}{l}i=k \\
a_{k}\end{array}$ & & & $4 \beta^{4} k$ & & $2 \beta^{2}(2 k+1)$ & & $4 \beta^{4}$ \\
\hline $\begin{array}{l}i=k-1 \\
a_{k-1}\end{array}$ & & $4 \beta^{4} k(k-1)$ & & & & $4 \beta^{4} k$ & \\
\hline $\begin{array}{l}i=k-2 \\
a_{k-2}\end{array}$ & & & & & $4 \beta^{4} k(k-1)$ & & \\
\hline
\end{tabular}

The nonzero elements of the matrix $W^{(k)}$. 
Proof: We substitute (11) and (12) into (8.1), make use of (10.2), (10.1) and the formula

$$
x^{2} \widetilde{H}_{k}(x)=(k+1)(k+2) \tilde{H}_{k+2}(x)+\frac{2 k+1}{2 \beta^{2}} \widetilde{H}_{k}(x)+\frac{1}{4 \beta^{4}} \widetilde{H}_{k-2}(x), \quad k=2,3, \ldots
$$

which is obtained from (10.1).

After equating the coefficients at $\tilde{H}_{k}(x), k=0,1, \ldots, n-2$ and multiplying the obtained equations by $4 \beta^{4}$ we come to the system

$$
\begin{aligned}
c_{1} a_{k+3} & +\left(2 \beta^{2} c_{2}+c_{4}-4 \beta^{4} \varepsilon^{2}\right) a_{k+2}+2 \beta^{2}\left((2 k+1) c_{1}+2 \beta^{2} c_{3}+c_{5}\right) a_{k+1} \\
& +2 \beta^{2}\left(2 \beta^{2} k c_{2}+(2 k+1) c_{4}+2 \beta^{2} c_{6}\right) a_{k}+4 \beta^{4}\left(k(k-1) c_{1}+k c_{5}\right) a_{k-1} \\
& +4 \beta^{4} k(k-1) c_{4} a_{k-2}=4 \beta^{4} b_{k}, \quad k=0,1, \ldots, n-2 .
\end{aligned}
$$

(For $k=1$ the last term is omitted and for $k=0$ the last two terms are omitted.)

The obtained equalities are obviously of the form (13.1). The last two equations (13.2) are obtained directly, by substituting (11) into the boundary conditions (8.2). The values for $\widetilde{H}_{k}(1-\delta)$ and $\widetilde{H}_{k}(1)$ are evaluated recurrently using the formula (10.1) in the form

$$
\widetilde{H}_{k+1}(x)=\frac{1}{k+1}\left(x \widetilde{H}_{k}(x)-\frac{1}{2 \beta^{2}} \widetilde{H}_{k-1}(x)\right), \quad k=1,2, \ldots, \widetilde{H}_{0}(x)=1, \quad \widetilde{H}_{1}(x)=x
$$

After the system (13.1), (13.2) is solved for $a_{k}, k=0, \ldots, n$, the value of the approximate solution (11) at the point $x \in[1-\delta, 1]$ can be evaluated by the use of the following algorithm:

$$
\begin{cases}\text { let } & \ell_{n+2}=\ell_{n+1}=0 \\ \text { let } & \ell_{n-k}=a_{n-k}+\frac{x}{n-k+1} \ell_{n-k+1}-\frac{1}{2 \beta^{2}(n-k+2)} \ell_{n-k+2}, \quad k=0, \ldots, n \\ \text { let } & u_{n}(x)=\ell_{0} .\end{cases}
$$

\section{THE ERROR ESTIMATE}

Out of the boundary layer, the exact solution of the problem (1.1), (1.2) is approximated by the solution of the reduced problem. It is well known (see for example [1]) that the following estimate is valid

$$
d(x)=\left|y(x)-y_{r}(x)\right| \leqslant C\left(\varepsilon^{2}+\exp \left(-F \delta / \varepsilon^{2}\right)\right), \quad x \in[0,1-\delta]
$$

Throughout the paper $C$ will denote an arbitrary constant independent of $x$ and $\varepsilon$. 
Let us, now, estimate the error upon the layer subinterval $[1-\delta, 1]$. The error function, according to (4.1) and (4.2) is

$$
d(x)=\left|y_{\varepsilon}(x)-u_{n}(x)\right| .
$$

In order to estimate it we first have to prove the following lemma:

Lemma 3. let $x \in[1-\delta, 1]$. Then

$$
\left|y_{e}(x)-u(x)\right| \leqslant d_{0}, \quad d_{0}=C\left(\varepsilon^{2}+\exp \left(-F \delta / \varepsilon^{2}\right)\right) .
$$

Proof: The function $y_{\varepsilon}(x)$ satisfies the boundary value problem

$$
\begin{aligned}
& L y_{e} \equiv-\varepsilon^{2} y_{\varepsilon}^{\prime \prime}(x)+f(x) y_{e}^{\prime}(x)+g(x) y_{e}(x)=\varepsilon^{2} y_{r}^{\prime \prime}(x), \quad x \in[1-\delta, 1] \\
& D y_{\varepsilon} \equiv\left(y_{e}(1-\delta), y_{\varepsilon}(1)\right)=\left(y(1-\delta)-y_{r}(1-\delta), B^{0}\right) .
\end{aligned}
$$

Subtracting (4.3), (4.4) from (17.1), (17.2) we obtain

$$
L\left(y_{\varepsilon}-u\right)=0, \quad D\left(y_{\varepsilon}-u\right)=\left(y(1-\delta)-y_{r}(1-\delta), 0\right) .
$$

By the principle of inverse monotonicity we can conclude that

$$
\left|y_{\varepsilon}(x)-u(x)\right| \leqslant\left|y(1-\delta)-y_{r}(1-\delta)\right| .
$$

Using the estimate (14) for $x=1-\delta$ we obtain (16).

Before we prove the following theorem we have to give one more lemma.

Lemma 4. Assume that for two functions $v, w \in C^{2}[1-\delta, 1]$ the following inequalities hold

$$
v \leqslant w, \quad L v \leqslant 0 \leqslant L w, \quad D v \leqslant\left(0, B^{0}\right) \leqslant D w .
$$

Then the problem (4.3), (4.4) has a solution $u \in C^{2}[1-\delta, 1]$ with

$$
v \leqslant u \leqslant w .
$$

This lemma is a special case of $L 6$, which was proved by Lorenz in [6].

THEOREM 2. Let $v(x)$ and $w(x)$ be the exact solutions of the problems

$$
\begin{aligned}
& -\varepsilon^{2} v^{\prime \prime}(x)+F_{2} v^{\prime}(x)+K_{2}^{2} v(x)=0, \quad x \in[1-\delta, 1], v(1-\delta)=0, v(1)=B^{0} \\
& -\varepsilon^{2} w^{\prime \prime}(x)+K_{1}^{2} w(x)=0, \quad x \in[1-\delta, 1], \quad w(1-\delta)=0, w(1)=B^{0}
\end{aligned}
$$


where $F_{2}, K_{1}, K_{2} \in R$ are such constants that $f(x) \leqslant F_{2}, K_{1}^{2} \leqslant g(x) \leqslant K_{2}^{2}$, while $x \in[1-\delta, 1]$ and

$$
d_{n}(x)=\max \left\{\left|v(x)-u_{n}(x)\right|, \quad\left|w(x)-u_{n}(x)\right|\right\} .
$$

Then the error $d(x)$ defined by (15) can be estimated as

$$
d(x) \leqslant d_{0}+d_{n}(x), \quad x \in[1-\delta, 1],
$$

where $d_{0}$ is defined in (16).

Proof: We can see that

$$
d(x) \leqslant\left|y_{\epsilon}(x)-u(x)\right|+\left|u(x)-u_{n}(x)\right|,
$$

where $u(x)$ is the solution of the problem (4.3), (4.4).

Let us, first, assume that $B^{0}=y(1)-y_{r}(1)>0$. Then, applying the principle of inverse monotonicity to the problems (18.1) and (18.2), we have $v(x) \geqslant 0$ and $w(x) \geqslant 0$. We also have $v^{\prime}(x) \geqslant 0, w^{\prime}(x) \geqslant 0$ for $x \in[1-\delta, 1]$. We can define the functions $f_{2}(x) \geqslant 0, g_{1}(x) \geqslant 0, g_{2}(x) \geqslant 0$, such that $f(x)=F_{2}-f_{2}(x) . g(x)=K_{1}^{2}+g_{1}(x)$ and $g(x)=K_{2}^{2}-g_{2}(x)$ for $x \in[1-\delta, 1]$. Then, using (18.1) and (18.2), we obtain

$$
\begin{aligned}
L v & =-\varepsilon^{2} v^{\prime \prime}(x)+\left(F_{2}-f_{2}(x)\right) v^{\prime}(x)+\left(K_{2}^{2}-g_{2}(x)\right) v(x) \\
& =-f_{2}(x) v^{\prime}(x)-g_{2}(x) v(x) \leqslant 0
\end{aligned}
$$

$$
L w=-\varepsilon^{2} w^{\prime \prime}(x)+f(x) w^{\prime}(x)+\left(K_{1}^{2}+g_{1}(x)\right) w(x)=f(x) w^{\prime}(x)+g_{1}(x) w(x) \geqslant 0
$$

and

$$
\begin{aligned}
& -\varepsilon^{2}(v-w)^{\prime \prime}(x)+F_{2}(v-w)^{\prime}(x)+K_{2}^{2}(v-w)(x) \\
& =-F_{2} w^{\prime}(x)-\left(K_{2}^{2}-K_{1}^{2}\right) w(x) \leqslant 0, \quad x \in[1-\delta, 1]
\end{aligned}
$$

$$
(v-w)(1-\delta)=0, \quad(v-w)(1)=0
$$

By the principle of inverse monotonicity from (22.3), (22.4) the following inequality is valid:

$$
v(x)-w(x) \leqslant 0 \quad \text { for } \quad x \in[1-\delta, 1]
$$

Finally,

$$
D v=D u=D w=\left(0, B^{0}\right)
$$


Now, according to (23), (22.1), (22.2) and (24), we can apply the result of Lemma 4, which gives us

$$
v(x) \leqslant u(x) \leqslant w(x), \quad x \in[1-\delta, 1] .
$$

After subtracting $u_{n}(x)$ in the above inequality we can conclude that

$$
u(x)-u_{n}(x) \leqslant \max \left\{\left|v(x)-u_{n}(x)\right|,\left|w(x)-u_{n}(x)\right|\right\}=d_{n}(x) .
$$

In the case $B^{0}<0$, using the same technique, we again arrive at (25). Finally, using the estimates (16) and (25) in (21), we obtain (20).

REMARK. The same technique can be applied in solving self adjoint boundary layer problems.

\section{NUMERICAL RESUlTS}

We shall use the following test example

$$
\begin{gathered}
-\varepsilon^{2} y^{\prime \prime}(x)+\frac{1-\varepsilon}{(1+x)^{2}} y(x)=\frac{(\varepsilon-1) x}{(1+x)^{2}} \quad x \in[0,1] \\
y(0)=0 . \quad y(1)=0,
\end{gathered}
$$

whose solution is

$$
y(x)=\frac{1-(1+x)^{-2 /(1+\varepsilon)}}{1-2^{-2 /(1+\varepsilon)}}\left(\frac{1+x}{2}\right)^{1 / e}-x .
$$

This problem was considered in [1].

The solution of the reduced problem is $y_{r}(x)=-x$, so that we have one boundary layer at $x=1$. The numerical layer length, applying (6.1), is

$$
\delta=2 \varepsilon \sqrt{\frac{n(n-1)}{1-\varepsilon}}
$$

In the following tables we shall give the values of the exact solution, the error $d(x)$ and the error estimate (20) in several points from the boundary layer for $n=4$ and $\varepsilon=10^{-5}\left(10^{-7}\right)$. We have chosen $\beta=2 / \delta$ in $(9.1)$.

Table 2.

\begin{tabular}{|l|c|c|c|}
\hline$\varepsilon=10^{-5}$ & \multicolumn{2}{|c|}{$n=4$} & \multicolumn{2}{c|}{$\delta=6.93 \cdot 10^{-5}$} \\
\hline \multicolumn{1}{|c|}{} & $y(x)$ & $d(x)$ & $d_{0}+d_{n}(x)$ \\
\hline 0.99996 & -0.86 & $4.8(-2)$ & $5.6(-2)$ \\
0.99997 & -0.78 & $1.0(-2)$ & $1.4(-2)$ \\
0.99998 & -0.63 & $3.4(-2)$ & $3.2(-2)$ \\
0.999987 & -0.48 & $5.0(-2)$ & $4.9(-2)$ \\
0.99999 & -0.39 & $4.9(-2)$ & $4.8(-2)$ \\
0.999994 & -0.26 & $3.9(-2)$ & $3.8(-2)$ \\
0.999997 & -0.14 & $2.3(-2)$ & $2.3(-2)$ \\
0.999999 & -0.05 & $8.6(-3)$ & $8.5(-2)$ \\
\hline
\end{tabular}


Table 3.

\begin{tabular}{|c|c|c|c|}
\hline \multicolumn{1}{|c}{$\varepsilon=10^{-7}$} & \multicolumn{2}{c|}{$\delta=6.93 \cdot 10^{-7}$} \\
\hline$x$ & $y(x)$ & $d(x)$ & $d_{0}+d_{n}(x)$ \\
\hline 0.9999996 & -0.86 & $4.9(-2)$ & $5.6(-2)$ \\
0.9999997 & -0.78 & $9.8(-3)$ & $1.4(-2)$ \\
0.9999998 & -0.63 & $3.5(-2)$ & $3.2(-2)$ \\
0.99999987 & -0.48 & $5.0(-2)$ & $4.9(-2)$ \\
0.9999999 & -0.39 & $4.9(-2)$ & $4.8(-2)$ \\
0.99999994 & -0.26 & $3.9(-2)$ & $3.8(-2)$ \\
0.99999997 & -0.14 & $2.4(-2)$ & $2.3(-2)$ \\
0.99999999 & -0.05 & $9.5(-3)$ & $8.5(-3)$ \\
\hline
\end{tabular}

\section{ReFerences}

[1] E.P. Doolan, J.J.H. Miller and W.H.A. Schilders, Uniform numerical methods for problems with initial and boundary layers (Dublin Boole Press, 1980).

[2] T.S. Horner, 'Recurrence relations for the coefficients in Chebyshev series solution of ordinary differential equations', Math. Comp. 35 (1980), 893-905.

[3] R.Y. Chang and M.L. Wang, 'The properties of modified Hermite polynomials and their applications to functional differential equations', J. Chinese Inst. Engrs. 9 (1986), 75-81.

[4] N. Adžić, 'Recurrence relations for the coefficients in Legendre solution for some ordinary differential equations', Univ. u Novom Sadu Zb. Rad. Prirod.-Mat. Fak. Ser. Mat. 19 (1988), 14-25.

[5] N. Adžić, 'On orthogonal series solution for boundary layer problems', Publ. Inst. Math. Univ. Nancago 49 (63) (1990) (to appear).

[6] J. Lorenz, 'Stability and monotonicity properties of stiff quasi-linear boundary problems', Univ. u. Novom Sadu Zb. Rad. Prirod.-Mat. Fak. Ser. Mat. 12 (1982), 151-173.

Faculty of Technical Sciences

Institute for Appl. Fund. Disc.

Veljka Vlahovića 3

21000 Novi Sad

Yugoslavia 